\title{
Cutaneous ulceration in Crohn's disease
}

J. C. MOUNTAIN ${ }^{1}$

From St Mark's Hospital, London

SUMMARY This paper describes in detail seven cases of extensive perineal ulceration seen in 207 cases of Crohn's disease at St Mark's Hospital in the last eight years. Four of these cases also had cutaneous ulceration elsewhere. Suggestions are made for the management of further cases of this rare condition.

Extensive cutaneous ulceration in Crohn's disease is a rare complication which has become clinically apparent in the last few years.

It is well known that anal lesions are common manifestations, occurring in approximately $25 \%$ of ileal and $80 \%$ of large intestinal Crohn's disease, and they may precede the abdominal condition by many years (Lockhart-Mummery and Morson, 1964). The clinical features and pathology of these anal lesions have been described in detail in previous reports from this hospital (Morson and Lockhart-Mummery, 1959; Gray, Lockhart-Mummery, and Morson, 1965).

It has been mentioned that gross perineal ulceration occasionally occurs, leading to extensive destruction of the anal sphincters and surrounding tissue but it has never been described in detail, except for three isolated cases reported to the Royal Society of Medicine, including case 4 of our own series (Hunter-Craig 1961; Scott, 1961; Parks, Morson, and Pegum, 1965).

Cutaneous ulceration, other than in the perineal region, does not appear to be well documented in the literature, although it was mentioned by McCallum and Kinmont in 1968, in a review of the dermatological manifestation of Crohn's disease. In reviewing the 207 cases of Crohn's disease seen in the last eight years at St Mark's Hospital, seven cases of extensive perineal ulceration were

Present address: Royal Victoria Hospital, Boscombe, Bournemouth found and four of these had cutaneous ulceration elsewhere. They are now presented as they all demonstrate different features of this condition.

\section{Case Histories}

\section{CASE 1}

V.K., a girl aged 14, was referred to St Mark's Hospital in June 1961 with gross perineal ulceration. There was a history of perineal soreness in 1957 when 10 years of age, which settled with local caladryl and saline baths. In 1959 she developed gross oedema and inflammation of the labia and an anal fistula. A biopsy was performed which showed chronic low-grade inflammatory changes but no tubercles or caseation; however, the patient was given a two-month course of streptomycin and Inah without benefit.

Sigmoidoscopy in October 1959 revealed multiple granular nodules in the rectum but a barium enema was reported as normal. Treatment with systemic prednisolone was begun and continued for 18 months. This resulted in improvement but healing was incomplete. A further biopsy in the anal region in 1961 showed multinucleated giant cells of the tuberculous type and it was considered that the lesion was a tuberculide of the tuberculous sarcoid group. 
When she was admitted to St Mark's Hospital in June 1961 there was extensive ulceration of the perineal skin. The previous histological sections were reviewed by Dr Morson and he considered the changes to be consistent with a diagnosis of Crohn's disease. A defunctioning ileostomy without resection of the colon was performed. The appendix, which was removed during this operation, showed non-caseating giant cell and epithelioid cell systems supporting the diagnosis of Crohn's disease. The anal ulceration initially improved but eventually relapsed.

In August 1963 a synchronous combined excision of the rectum and lower sigmoid colon was performed and the defunctioning ileostomy closed. Thereafter the perineal wound healed well but the skin around the colostomy became desquamated and subsequently ulcerated (Fig. 1). By July 1965 gross ulceration was present around the colostomy and a biopsy confirmed the presence of Crohn's disease. A barium enema showed narrowing with fissuring of the last few centi-

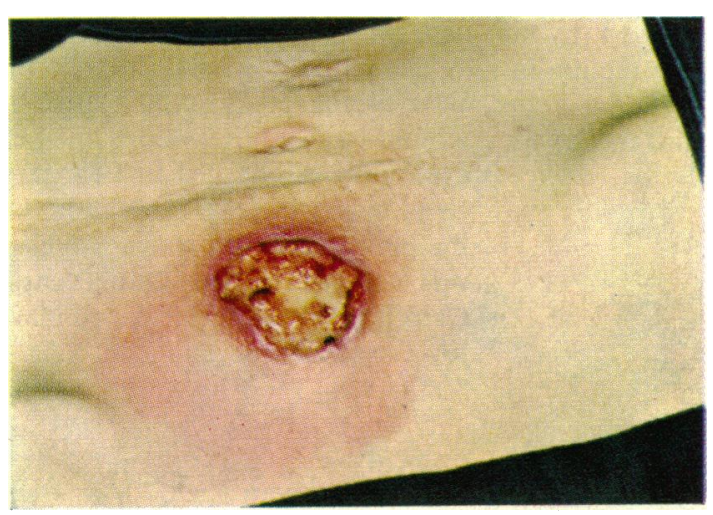

Fig. 1 Paracolostomy ulceration in case 1 .

metres of the colon consistent with a recurrence of the disease in this segment.

In August 1965 the remainder of the colon was excised and a permanent ileostomy established. Histology of the excised specimen showed Crohn's disease of the distal colon with early lesions in the ileo-caecal region, the right colon, and the transverse colon.

The postoperative course was satisfactory and the ulcerated area on the abdomen healed rapidly with the aid of skin grafts. She was last seen in July 1968 when she was very well with no ulceration of the perineum or abdominal wall.

CASE 2

A.S., a man aged 54, was referred to St Mark's Hospital in August 1963. There was a history of a rectal discharge developing in July 1961 which continued until a complicated fistula was laid open in October 1962.
Postoperatively the wound showed no signs of healing and his general condition remained very poor, with general malaise and continuous rectal discharge. Sigmoidoscopy in February 1963 showed inflammation of the lower rectal mucosa and a biopsy revealed non-specific inflammation. As his general condition did not improve an ileostomy was performed in April 1963 after which he made good progress for about two months only to relapse again with malaise, perineal discharge, and fluid ileostomy actions.

The only other relevant history was a traumatic rupture of the bulbous urethra 25 years previously.

On examination he was in poor general condition, being anaemic and obviously having lost considerable weight. A loop ileostomy appeared satisfactory but there was gross undercutting ulceration of the perineum extending onto both sides of the scrotum anteriorly, into the ischiorectal fossae laterally, and as far as the sacrum posteriorly. It was impossible to determine the site of the anus on initial examination as this also was ulcerated.

A chest radiograph was normal, a barium enema was impossible. Culture of pus from the perineum revealed no tubercule bacilli. He was examined under an anaesthetic. Sigmoidoscopy to $14 \mathrm{~cm}$ revealed an ulcerated oedematous rectal mucosa with complete desctruction of the anal canal and loss of the anal sphincter. Biopsy of the anal canal region confirmed Crohn's disease though rectal biopsy showed non-specific inflammatory changes only.

In September 1963 a proctocolectomy, but preserving the old ileostomy, was performed. At operation the whole colon was involved with Crohn's disease and this was confirmed on histological examination of the specimen. Postoperative progress was extremely stormy and the perineal wound showed few signs of healing. Three months after the initial operation he suddenly developed an acute abdomen which at laparotomy proved to be due to a spontaneous rupture of the spleen and splenectomy was performed. Afterwards his progress was satisfactory and he was discharged one month later though the perineum remained unhealed.

In July 1965 the ulceration had again extended and he was admitted and the wound curetted. Histology of the curettings confirmed the diagnosis of Crohn's disease. No form of local dressing appeared to produce any improvement in the ulceration and the wound was again curetted in March 1966.

In July 1966 he developed a spontaneous perineal urinary fistula through the bulbous urethra which was confirmed by urethrogram. Synalar N cream was applied to the perineum at this time with no effect and in November 1966 he was started on oral prednisone which was continued until July 1967.

In November 1967 he was admitted again for 
reassessment with persistent perineal ulceration and urinary fistula. As neither condition was causing him any concern and he was generally very well no operative treatment was advised but he was started on bacitracin insufflations to the wound.

He has not attended for follow up since this admission.

\section{CASE 3}

L.W., a man aged 33, was admitted to St Mark's Hospital in September 1963 with an unhealed perineal wound. There was a history of a perineal abscess being laid open in 1958 after which he developed diarrhoea severe enough to warrant treatment with steroids. The diarrhoea recurred in 1959 but responded to a period of medical treatment in hospital.

In February 1960 he developed an ischiorectal abscess and associated fistula which was laid open but diarrhoea returned on decreasing the oral steroids.

A colectomy and ileostomy was performed in September 1961 and this was followed by a synchronous combined excision of the rectum in May 1962.

On admission to St Mark's Hospital there was

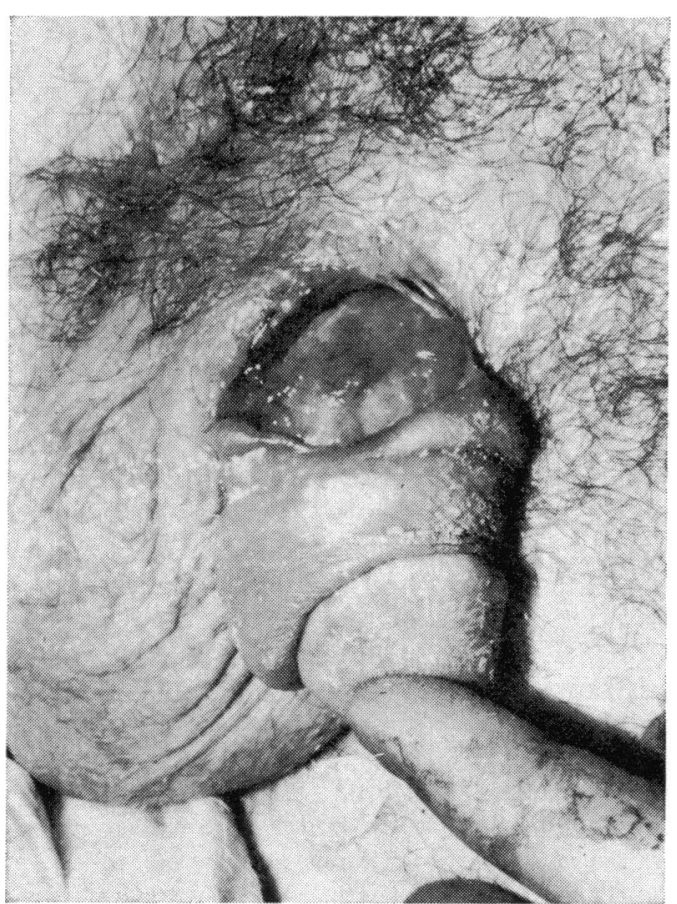

Fig. 2 Metastatic ulceration at the base of the penis, in case 3. some ulceration of the angles of the mouth and face creases and he had a typical moon face due to steroid therapy. Abdominal examination revealed ulceration and excoriation of the skin around the ileostomy but otherwise there was no abnormality. There was, however, gross ulceration of the perineum extending round the root of the scrotum into the right groin. A separate ulcer at the base of the penis with a subcutaneous fistula to the coronal region was also present (Fig. 2).

He was examined under an anaesthetic and a biopsy of the perineum taken. This showed sarcoid foci typical of Crohn's disease and later a biopsy was taken of the para-ileostomy ulceration and this again confirmed Crohn's disease. A Mantoux test $(1-1,000)$ was positive but no acid alcohol-fast bacilli were found in the curettings and guinea-pig inoculation was negative.

Repeated curettage was performed in the next four months and a variety of local applications were tried, including potassium permanganate dressings and Savlon dressings and also various types of steroid lotions. The oral steroids were also gradually tailed off.

The para-ileostomy ulceration and ulcer at the base of the penis soon healed but the perineal wound was rather slow in healing. He was eventually discharged in February 1964. He was re-admitted in July 1964 and March 1965 when the perineal wound was again curetted. When he was last seen in the Outpatient Department in October 1968 the perineal wound was healed except for a small sinus.

\section{CASE 4}

M.Y., a woman aged 70 , was transferred to $\mathrm{St}$ Mark's Hospital with an unhealed perineal wound in October 1963.

In 1959 she developed diarrhoea with blood and mucus which was diagnosed as being due to ulcerative colitis and responded to a period of bed rest in hospital and oral steroids. After discharge from hospital her condition again deteriorated and she eventually had a total colectomy and ileostomy performed in 1961. Pathological examination of the specimen confirmed the diagnosis of Crohn's disease.

Subsequent progress was satisfactory except for a discharging fistulous track in the paraumbilical region, until December 1962 when she developed acute intestinal obstruction which subsided conservatively. As she was also passing large quantities of blood from the rectum a synchronous combined excision was performed in January 1963. The immediate postoperative progress was satisfactory but the perineal wound remained unhealed.

On admission to St Mark's Hospital the perineal ulceration extended into the fourchette on either side of the labia majora and into the groins, and a biopsy showed the typical sarcoid 
reaction of Crohn's disease. Right submammary 'intertrigo' and eczema of both legs were noted at this time but were not biopsied.

A chest radiograph showed no evidence of active disease and a Mantoux test was negative to 1 in 10,000 but positive to 1 in 1,000 .

Initially only local treatment, including steroid applications, was given but after one month the perineal wounds were curetted and a skin graft was applied. This did not take and she was therefore put on prednisone, $40 \mathrm{mg}$ daily, with fairly rapid improvement. She was eventually discharged in January 1964 when the prednisone had been reduced to $15 \mathrm{mg}$ daily.

As soon as she returned home the condition relapsed so the prednisone dosage was again increased with some improvement but it had deteriorated again by May 1964 so she was readmitted. Fresh biopsies confirmed the diagnosis of Crohn's disease. After further local treatment she was discharged in June only to be admitted again in September. The perineal ulceration had now extended on both sides of the labia majora further into the groins and there was a large ulcer $76.2 \mathrm{~mm} \times 12.7 \mathrm{~mm} \mathrm{(3} \mathrm{in.} \times \frac{1}{2}$ in.) and in the right submammary region. A biopsy of this ulcer showed 'chronic inflammation with a florid sarcoid reaction consistent with Crohn's disease'. Further investigations to exclude tuberculosis again proved negative and examination of the ileostomy showed a normal mucosa to $10 \mathrm{~cm}$.

Though she was anaemic with a haemoglobin

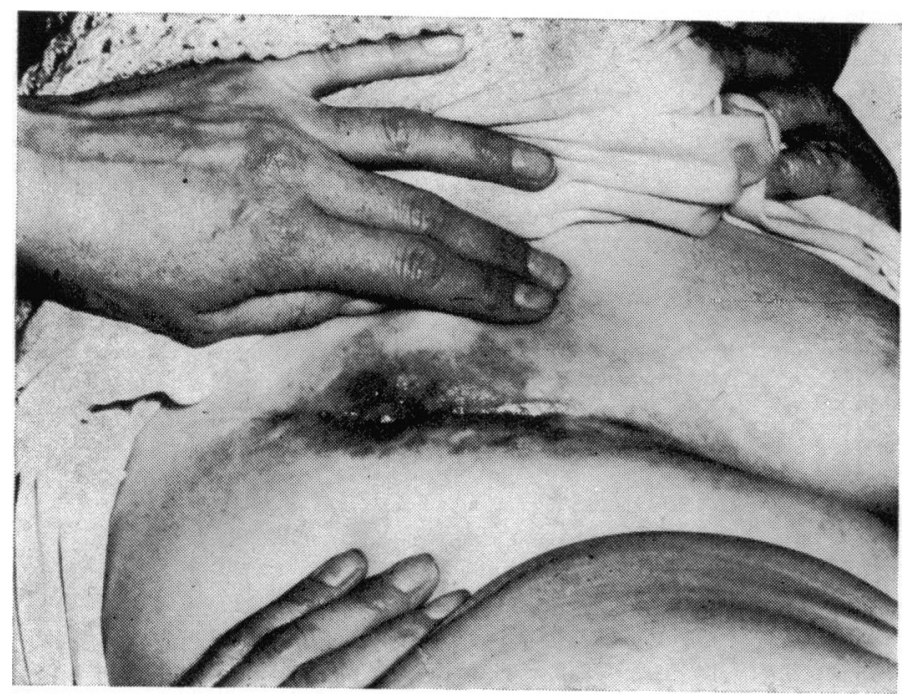

Fig. 3 Metastatic ulceration in the submammary region in case 4. of $64 \%$ it was decided to treat her with cytotoxic agents. After an initial blood transfusion she was started on 6 mercaptopurine $100 \mathrm{mg}$, tds, which was gradually reduced to $25 \mathrm{mg}$ tds. As there was no improvement on this regime she was also put back on prednisone, $20 \mathrm{mg}$ daily, in October 1964 and she gradually improved, with complete healing of the right submammary lesion over the next four weeks. In January 1965 the 6 mercaptopurine was replaced by Imuran, $150 \mathrm{mg}$ daily, and this was eventually stopped in March 1965.

Over the first six months of 1965 there was very little improvement in the perineal and groin wounds despite intermittent curettage and a great variety of local dressings. In March 1965 the right groin wound was excised and closed by primary suture but this broke down within two weeks.

She was eventually discharged in May 1965, and has been followed up regularly as an outpatient since then, and, though her general health has remained quite good, the ulceration persists despite all form of treatment.

\section{CASE 5}

J.D., a man aged 67, was transferred to St Mark's Hospital in November 1966 from a sanatorium where he was being treated as a case of anal tuberculosis. He had been previously diagnosed elsewhere as a case of 'ulcerative colitis' in 1958 and of 'diverticulitis' in 1960 . He remained relatively well until 1965 when he developed an anal fistula which failed to heal in spite of treatment. In that year he had a left iliac colostomy and subsequently a right transverse colostomy as the former was unsatisfactory but the fistula still did not heal. In June 1966 a biopsy from the edge of the fistula was reported as tuberculous granulation tissue and the patient was therefore transferred to the sanatorium and treated with antituberculous drugs for four months without effect.

On admission to St Mark's Hospital the patient's general condition was very poor, as he had lost over $4 \frac{1}{2}$ stone in weight. A Devine type transverse colostomy was functioning normally but there was a purulent discharge from the left iliac loop colostomy. There was gross ulceration of the perianal region and perineum extending forwards to involve the scrotum and both groins (Figs 4 and 5) and exposing the hamstrings and pelvic floor muscles. A blind sinus was also noted on the dorsum of the penis along which a probe could be passed for over 1 inch $(25.4 \mathrm{~mm})$. A barium enema via the colostomies showed the distal part of the transverse colon, the splenic flexure, the descending and the sigmoid colon to be grossly irregular, with narrowing and ulceration consistent with Crohn's disease, and this was. confirmed on sigmoidoscopy. A barium meal and follow through showed no lesion in the small bowel. 

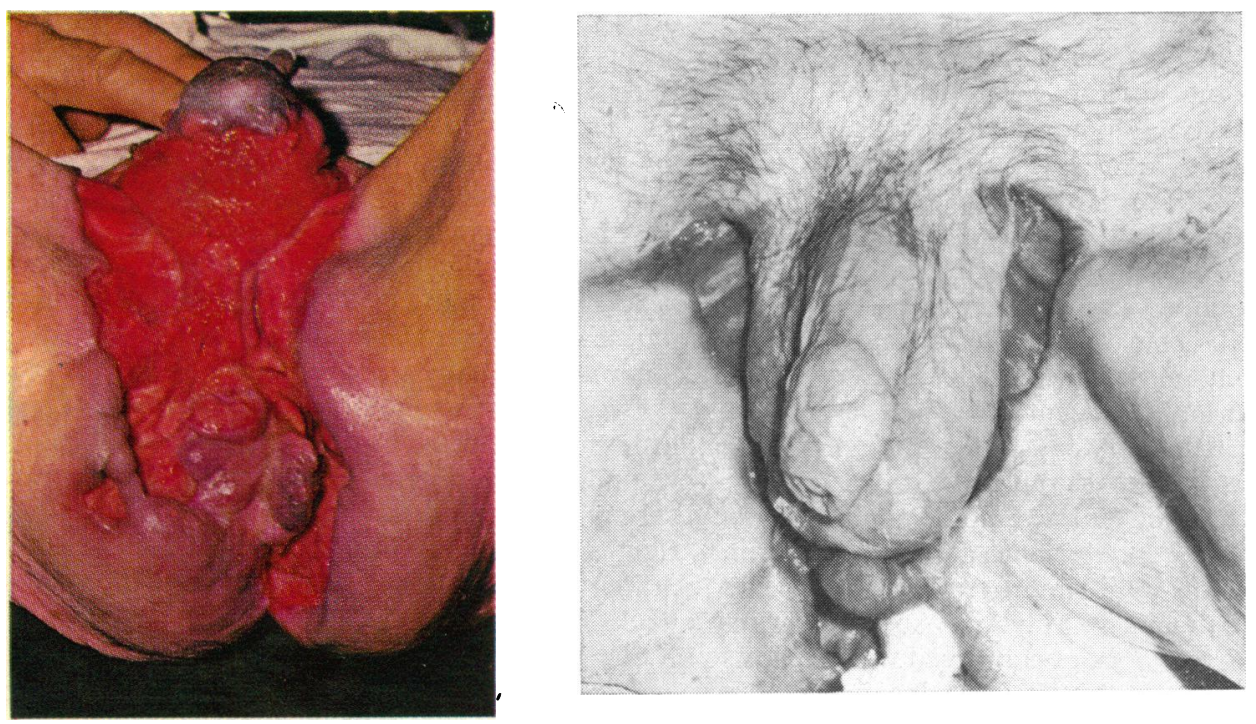

Fig. 4 Perinerl ulceration extending into the groins in case 5 (left).

Fig. 5 Perineal ulceration extending into the groins in case 5 (right).

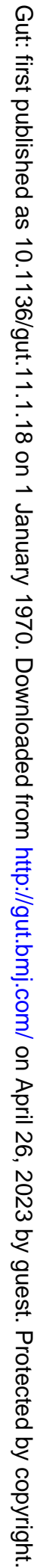

Fig. 6 Typical follicular lesions under the perineal skin in case $5 \times 20$. 


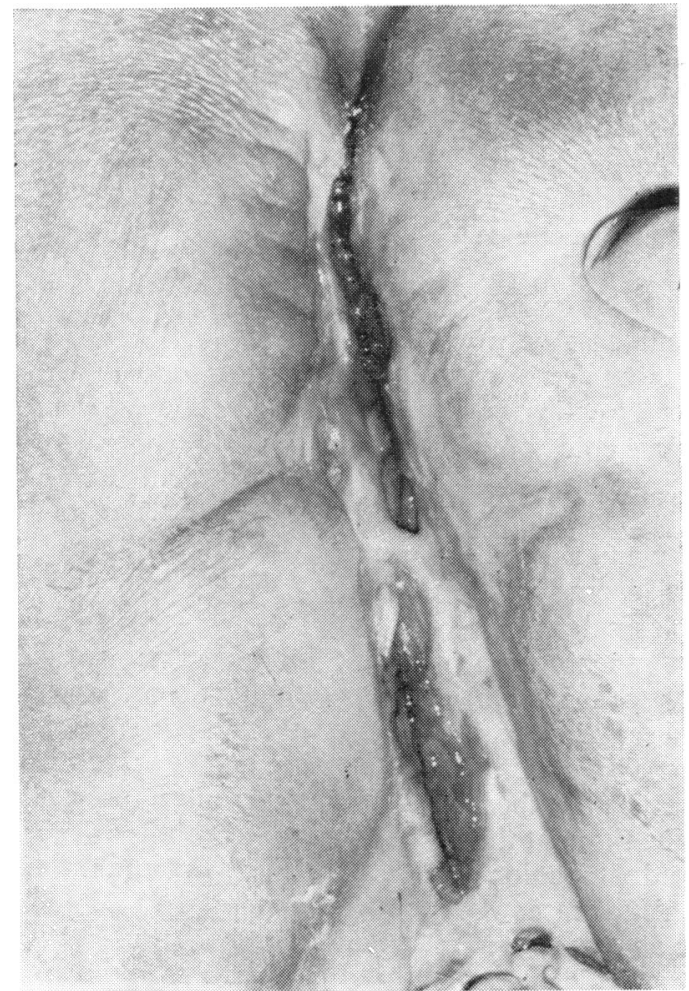

Fig. 8 Eleven months after proctocolectomy in case 5 .

The patient's general condition improved on intensive supportive therapy and a total proctocolectomy was performed in December 1966. Pathological examination of the excised specimen confirmed Crohn's disease of the colon, rectum, anal canal, and perianal region (Figs 6 and 7).

The postoperative course was complicated by a left lower lobe collapse, urinary infection, and an abdominal wound abscess but subsequently his progress was steady and the perineal wound, which was initially enormous, gradually closed after the use of local potassium permanganate dressings and oral zinc sulphate, $200 \mathrm{mg}$ tds. The perineal wound was regularly reviewed under general anaesthesia and intermittent curettage was performed over the next five months. He was eventually discharged in May 1967 when the perineal wound was still $76.2 \mathrm{~mm}$ ( 3 in.) deep and dressings were continued by the district nurse.

His general condition continued to improve and he was readmitted in November 1967 when the perineal wound was curetted. At this time the left groin was completely healed and the right groin was almost healed but there was still a deep perineal cavity (Fig. 8). Histology of the curetting showed no sarcoid reaction.

In December 1968 he had gained over 4 stone in weight since his operation and although there was slight residual perineal ulceration it was not causing him any incapacity.
In November 1969 he developed a spontaneous urinary fistula which was demonstrated by urethrogram to be from the posterior urethra at the anterior part of the unhealed perineal wound. No treatment has been suggested as he is continent and therefore it does not cause him much inconvenience.

\section{CASE 6}

R.L., a man aged 46, was transferred to St Mark's Hospital with multiple ulcers and sinuses anterior to the anus extending into both groins in January 1967. In 1956-57 he had developed a high horseshoe fistula which had healed after multiple operations. There had been no further symptoms until July 1966 when he developed the ulceration and fistulae spontaneously. Sigmoidoscopy and a barium enema were completely normal. A provisional diagnosis of hidradenitis suppurativa with recurrent low fistula was made and the perianal skin was excised and primary skin grafting was performed in January 1967.

Histological sections taken from all parts of the excised specimen showed deep, penetrating and fissure-like ulcers surrounded by chronic inflammatory granulation tissue. There were numerous collections of epithelioid cells and giant cells, mainly of the Langhans type, in the form of a sarcoid reaction suggestive of a diagnosis of Crohn's disease. Postoperatively the skin graft did not take but the wound granulated well and healed slowly using Betnovate cream dressings. He was discharged in February 1967 and continued to dress the wound himself at home.

By August 1967 the wound had almost healed but in January 1968 the anal Crohn's disease relapsed and he developed a recurrent posterior horseshoe fistula and also a low anterior fistula associated with a new area of ulceration. He was readmitted and the anterior fistula was partially laid open to allow drainage of the wound but subsequently the ulceration extended anteriorly onto both sides of the scrotum. Excision of rectum was suggested to the patient at this time but he refused as he was able to manage the dressings of the extensive wound very well indeed and he was extremely fit and active.

The anal condition, however, continued to deteriorate so that by November 1968 the ulceration had extended both anteriorly and posteriorly. An abdomino-perineal excision was then performed and histology confirmed Crohn's disease confined to the anal region. He was discharged after six weeks when the perineum was not completely healed. He was last seen in March 1969 when healing was almost complete.

\section{CASE 7}

E.C., a woman aged 65 , was transferred to St Mark's Hospital in July 1967 with extensive cutaneous ulceration of the perineum, both groins, and in a transverse lower abdominal 
skin crease. There was a previous history of drainage of an ischio-rectal abscess in June 1958 followed by laying open of a fistula-in-ano in February 1960. After this operation she continued to pass pus from the rectum and a barium enema was reported as showing diverticulitis and ulcerative colitis. The symptoms subsided after a course of crystamycin.

She was readmitted in June 1960 with colicky abdominal pain and diarrhoea and passage of pus per rectum. The abdomen was tender over the colon and was distended. The fistula-in-ano was still discharging pus. A transverse colostomy was performed for subacute intestinal obstruction and an inflammatory sigmoid mass was noted.

In November 1960 the descending and sigmoid colon were resected with a clinical diagnosis of ulcerative colitis but the pathological report described patchy ulceration of the mucosa with multinucleated giant cells, probably of a foreign body type, near the ulcer bases and scattered foci of epithelioid cells, without caseation.The pathologist considered these features suggestive of tuberculosis but if this were disproved he thought the most likely diagnosis would be sarcoid of the colon; in fact this was certainly Crohn's disease. A Mantoux test was then performed and was positive. Though no definite diagnosis had so far been made she was started on streptomycin and Pasinah.

In February 1961 she was readmitted and a rectal stricture demonstrated from 5 to $10 \mathrm{~cm}$, with a red, haemorrhagic, thickened, oedematous mucosa. There was extensive superficial ulceration at the anus in the right lateral quadrant and this was excised. Histology of the specimen was reported as tuberculous granulation tissue. Subsequent progress was poor and eventually an abdomino-perineal excision of the rectum was performed for persistent rectal stricture and purulent discharge in February 1962. Histology again was reported as showing 'tuberculosis'.

After this operation progress was very satisfactory with increase of 3 stone in weight during the following year. Superficial ulceration of the perineum, groins, and transverse abdominal skin folds, however, still persisted. In December 1966 she was readmitted and the ulceration biopsied. This showed features consistent with Crohn's disease of the skin. In February 1967 the ulcerated areas were excised and primary suture was performed but these broke down within two weeks.

On admission to St Mark's Hospital there was linear deep ulceration in both groins, being more extensive on the right side and extending into the perineum. There was also a small area of separate ulceration on the lower abdominal wall. The ulceration was biopsied and curetted and histology confirmed the presence of giant cells and epithelioid cells consistent with a diagnosis of Crohn's disease. Repeated curettage twice weekly was performed under a general anaesthetic and lotio rubra dressings were applied. She was also given oral zinc sulphate, $200 \mathrm{mg}$ tds, and this regime resulted in rapid healing of the wounds so that it was possible for her to be discharged from hospital after three weeks.

\section{Discussion}

Cutaneous ulceration in Crohn's disease is not restricted to any one age group or sex. There were four men and three women and the age of our patients at the time of onset of the ulceration ranged from 10 to 69 years, with a mean age of 48 years; three of the patients were over 60 years of age.

Macroscopically the ulceration has florid granulation tissue and there is marked destruction of the surrounding tissues. The skin edge is oedematous and there is a typical dusky cyanosis of the surrounding skin (Lockhart-Mummery, 1965).

Though the ulceration is usually associated with severe Crohn's disease of the gastrointestinal tract the condition has to be differentiated from tuberculosis by demonstrating the typical sarcoid granulomatous reaction in the absence of central caseation in a biopsy of the ulceration, together with a normal chest radiograph and a negative Mantoux test to a dilution 1 in 100 . If the Mantoux test is positive, and no tubercle bacilli have been demonstrated in the granulation tissue a guinea-pig is inoculated with fresh tissue from the ulcerated area. If this inoculation is negative it may have to be repeated. It is interesting that three of our patients (cases 1, 5. and 7) were initially considered to be suffering from tuberculosis but the diagnosis was later changed on review of the histological sections and clinical picture and when healing had not occurred with antituberculous therapy.

The cutaneous ulceration occurs in three different sites: (1) perineal ulceration, (2) parastomal ulceration, and (3) 'metastatic' ulceration.

\section{PERINEAL ULCERATION}

This is the commonest site and in all our patients was the initial site of the disease. It may extend widely in the perineum, into the groins, and even as far as the anterior abdominal wall.

\begin{tabular}{llllll}
\hline $\begin{array}{l}\text { Case } \\
\text { No. }\end{array}$ & Sex & $\begin{array}{l}\text { Age } \\
\text { (Yr.) }\end{array}$ & \begin{tabular}{l} 
Ulceration \\
\cline { 3 - 5 } Perineal
\end{tabular} & Parastomal 'Metastatic' \\
\hline $\mathbf{1}$ & F & $\mathbf{1 0}$ & + & + & - \\
$\mathbf{2}$ & M & $\mathbf{5 3}$ & + & - & - \\
$\mathbf{3}$ & M & $\mathbf{3 2}$ & + & + & + \\
$\mathbf{4}$ & F & $\mathbf{6 9}$ & + & - & + \\
$\mathbf{5}$ & M & $\mathbf{6 6}$ & + & - & - \\
$\mathbf{6}$ & M & $\mathbf{4 5}$ & + & - & - \\
$\mathbf{7}$ & F & $\mathbf{6 0}$ & + & - & + \\
\hline
\end{tabular}

Table I Types of cutaneous ulceration in Crohn's disease 
Marshak, Lindner, and Janowitz (1966) observed that large intestinal Crohn's disease only appeared to extend after surgical interference. It is therefore interesting that in this small series the perineal ulceration tended to become extensive after some form of anal surgery; thus in three of our patients it began after an abdomino-perineal excision for rectal and anal disease and in two others after anal fistulae had been laid open. In our other two patients, however, the ulceration began without any recent anal surgery, and it is interesting to note that these were the only two cases where associated rectal disease was not initially present. Case 1 , however, eventually had an abdomino-perineal excision for the anal canal and perineal ulceration and she developed severe Crohn's disease in the proximal colon two years later which required a total colectomy and ileostomy.

Case 6 has also recently had an abdominoperineal excision but as yet has not developed disease of the proximal bowel.

The cutaneous ulceration appears to be just as liable as the ulceration in the gastrointestinal tract to erode into the surrounding structures. This is demonstrated by cases 2 and 5 where spontaneous perineal urinary fistula developed three years after proctocolectomy and it still persists. Case 2 had a traumatic rupture of the bulbous urethra 25 years previously and presumably therefore this area of the urethra was fibrotic and weakened but there was no similar history in case 5 .

\section{PARASTOMAL ULCERATION}

Occasionally ulceration occurs on the anterior abdominal wall in continuity with an ileostomy or colostomy. It is usually associated with recurrent disease of the adjacent bowel and is typically demonstrated by case 1 where the cutaneous ulceration healed rapidly soon after the diseased bowel had been excised by total colectomy and ileostomy.

Case 3 probably developed para-ileostomy ulceration but it is interesting that in this patient ileal disease has never been demonstrated and this ulceration healed quickly with conservative measures. It should therefore probably be considered to be a second area of 'metastatic' ulceration similar to the ulcer at the base of the penis.

\section{METASTATIC ULCERATION}

This appears to be a very rare condition, and, other than a previous report of case 4 , we have only found this condition mentioned by McCallum and Kinmont (1968) when they stated that they had seen two cases of submammary ulceration but did not give any clinical details.

Ulceration is described as being metastatic when it is completely remote from the gastrointestinal tract and is, and always has been, separated from other areas of ulceration by normal intact skin. Three examples of this have been seen in this series. In case 3 there was an area of discrete ulceration on the anterior surface at the base of the penis. The para-ileostomy ulceration in this patient should also probably be considered to be metastatic. In case 4 there was submammary ulceration, and in case 7 there was an area of ulceration in a transverse crease on the lower abdominal wall.

In all these three cases it is interesting that the ulceration occurred in sites where there was close apposition of two skin surfaces and where the surfaces tended to become rather moist. In addition cases 4 and 7 were rather obese women, the type of person in whom intertrigo and similar skin lesions tend to occur. In all three cases, however, the typical sarcoid granulomatous reaction has been demonstrated on biopsy of the lesions. So far no explanation can be given for this interesting complication of Crohn's disease.

\section{TREATMENT}

It is difficult to discuss treatment of this rare condition due to the small number of cases and the variation in the extent of the ulceration but several basic principles have evolved during the care of these patients.

The first stage in treatment is usually the removal of the adjacent bowel if it is involved in the disease as the ulceration will never heal when the bowel is still present no matter what local treatment is employed. This statement may appear to contradict the earlier observation that usually anal surgical interference initially caused the ulceration to extend, but it is interesting that the only patient in the series who refused an abdominoperineal excision of rectum still had extensive ulceration of the perineum until he eventually agreed to surgery whereas in nearly all the other patients ulceration has gradually healed.

A simple diversion ileostomy had been performed in case 1 and a transverse colostomy in two patients (cases 5 and 7) but this did not result in any improvement in the ulceration. This confirms the findings of Jones, Lennard-Jones, and Lockhart-Mummery (1966) and it is therefore not a procedure which is recommended in the treatment of this condition.

Three attempts at excision and primary suture were made (cases 4,6 , and 7) but on each occasion the wounds broke down after about one week and this form of treatment should probably be abandoned.

Systemic steroids were given in four of the seven cases and though some improvement in the ulceration had been noted while the treatment was continued healing has not been complete and relapse has always occurred when the dosage was decreased.

Cytotoxic agents were used in case 4 following reports that their use sometimes resulted in a 
remission in ulcerative colitis (Bean, 1966, Bowen, Irons, Rhodes, and Kirsner, 1966) but there was no definite beneficial effect resulting from this therapy.

Various local applications to the ulceration were used, including Milton solution, potassium permanganate solution, lotio rubra, and various types of steroid lotions but there has been no evidence that one type of treatment is superior to the others.

Since Pories, Henzel, Rob, and Strain (1967) showed that oral zinc sulphate improved the healing rate of open pilonidal sinus wounds by $30 \%$ we have used this drug in the dosage of $200 \mathrm{mg}$ tds. So far two patients (cases 5 and 7) have been treated with this drug and the rapid healing may in some degree be due to this drug.

In my opinion more importantly, however, is repeated curettage. This is usually performed about every 10 days but may be done up to twice weekly with good effect. This is well illustrated by case 7 as she was transferred to St Mark's Hospital five and a half years after an abdomino-perineal resection still with gross ulceration of the perineum and groins. She was treated with a regime of oral zinc sulphate and repeated curettage and the wounds were almost healed within three weeks.
I wish to thank the consultant staff of St Marks' Hospital for permission to study their patients and also to publish this paper. This work was performed whilst receiving a grant from the St Mark's Hospital Research fund.

References

Bean, R. H. D. (1966) Treatment of ulcerative colitis with antimetabolites. Brit. med. J., 1, 1081-1084.

Bowen, G. E., Irons, G. V., Jr., Rhodes, J. B., and Kirsner, J. B. (1966). Early experiences with Azathioprine in ulcerative colitis. J. Amer. med. Ass., 195, 460-464.

Gray, B. K., Lockhart-Mummery, H. E., and Morson, B. C. (1965). Crohn's disease of the anal region. Gut, 6, 515-524.

Hunter-Craig, C. J. (1961). Granuloma of the groins and perineum secondary to chronic proctocolitis. Proc. roy. Soc. Med., $54,1019$.

Jones, J. H., Lennard-Jones, J. E., and Lockhart-Mummery, H. E. (1966). Experience in the treatment of Crohn's disease of the large intestine. Gut, 7, 448-452.

Lockhart-Mummery, H. E. (1965). Pathological lesions of the anal region associated with Crohn's disease. Dis. Colon and Rect., 8, 399-401.

- and Morson, B. C. (1964). Crohn's disease of the large intestine. Gut, 5, 493-509.

McCallum, D. I., and Kinmont, P. D. C. (1968). Dermatological manifestations of Crohn's disease. Brit. J. Derm., 80, 1-8.

Marshak, R. K., Lindner, A. E., and Janowity, H. D. (1966) Granulomatous ileocolitis. Gut, 7, 258-264.

Morson, B. C., and Lockhart-Mummery, H. E. (1959). Anal lesions in Crohn's disease. Lancet, 2, 1122-1123.

Parks, A. G., Morson, B. C., and Pegum, J. S. (1965). Crohn's disease with cutaneous involvement. Proc. roy. Soc. Med., 58, 241-242.

Pories, W. J., Henzel, J. H., Rob, C. G., and Strain, W. H. (1967). Acceleration of wound healing in man with zinc sulphate given by mouth. Lancet, 1, 121-124.

Scott, O. L. S. (1961). Granuloma of the groins and perineum secondary to chronic proctocolitis. Proc. roy. Soc. Med., 54,1019 . 